\title{
PERFORMANCE IMPACT OF MOBILITY IN AN EMULATED IP-BASED MULTIHOP RADIO ACCESS NETWORK
}

\author{
Philipp Hofmann, Christian Bettstetter, Jeremie Wehren*, \\ and Christian Prehofer \\ DoCoMo Euro-Labs, Future Networking Lab, Munich, Germany \\ lastname@docomolab-euro.com
}

\begin{abstract}
This paper investigates the performance of a multihop radio access network. In our testbed, nodes communicate to one access point using IEEE 802.11b and AODV routing. We measure the average packet delay and delivery ratio, if the node movement is emulated employing the random waypoint and random direction model, respectively. We find that random waypoint mobility yields up to $100 \%$ better results. This shows that the testbed performance is highly sensitive to the mobility model, even if comparable mobility behavior is assumed.
\end{abstract}

Keywords: Multihop radio access, routing performance, mobility modeling, testbed, ad hoc routing, AODV, random waypoint model, random direction model, network emulation.

\section{Introduction}

The paradigm of a multihop radio access network (MRAN) is that mobile nodes serve as "wireless routers" to extend the coverage of fixed access points (see Fig. 1). This new network architecture is especially interesting for mobile communication systems in which the cell size is very small, e.g., due to operation at high frequencies. Although the design of such networks is still in the research phase, basic functionalities can already be shown today through the interworking of IP-based ad hoc networking protocols and fixed IP networks [1-6].

This paper studies the packet-level performance of a single-cell MRAN, based on measurements in a WLAN testbed with one access

\footnotetext{
* Jeremie Wehren studies at the Institut Eurécom, Sophia-Antipolis, France, and Ecole Polytechnique Fédérale de Lausanne (EPFL), Switzerland.
} 


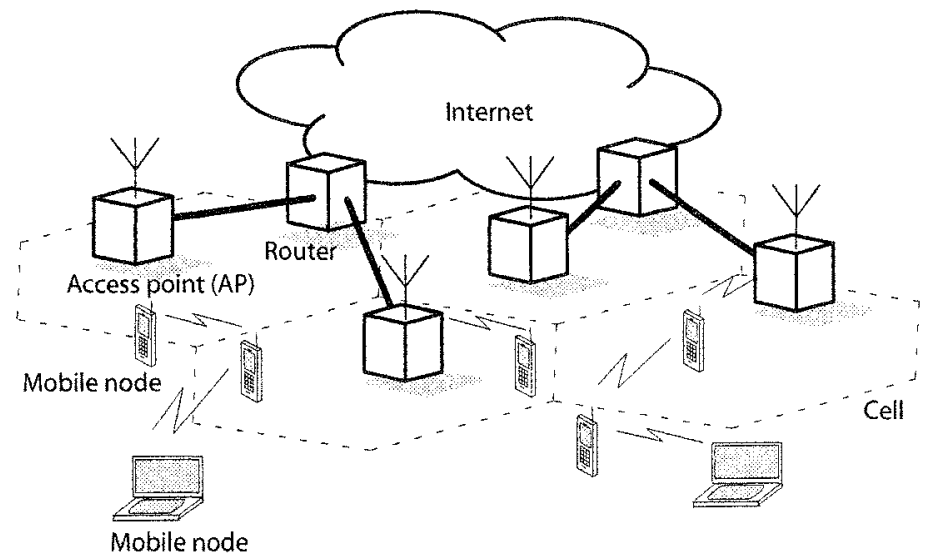

Figure 1. A multihop radio access network (MRAN) connected to a fixed network

point and seven mobile nodes. Each of the nodes runs the ad hoc ondemand distance vector (AODV) routing protocol [7] over IEEE $802.11 \mathrm{~b}$ in ad hoc mode. The mobile network is emulated by creating a virtual dynamic topology: the mobile nodes move virtually according to a given mobility model, and packets between nodes that are currently not within transmission range are filtered out.

We are especially interested in the impact of the mobility model on the network performance. To investigate this issue, we compare two wellknown models: the random waypoint (RWP) model [8] and the random direction (RD) model $[9,10]$. Although the two models are intuitively very similar, we show that the resulting performance is very different. Whereas the qualitative difference can be explained with recent theoretical results on both models [10-16], the quantitative difference is significant: using the RD model, the average packet delay is about twice as high as that of the RWP model; the packet delivery fraction of the RD model is almost $30 \%$ lower.

The remainder of this paper is organized as follows: Section 2 describes our testbed and emulation environment for MRANs. Section 3 explains the used mobility models and their inherent properties. Next, Section 4 presents and interprets the performance results. Section 5 addresses related work, before Section 6 concludes.

\section{Description of the Testbed}

As illustrated in Figure 2, our MRAN testbed consists of seven laptops and one desktop computer. The laptops represent the mobile nodes; the 


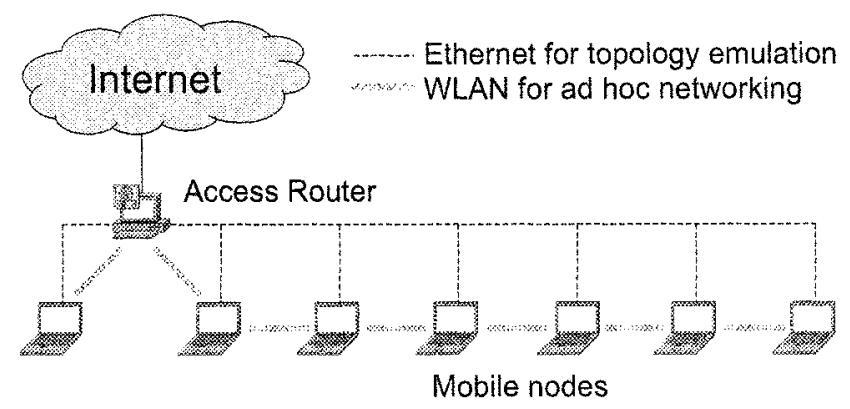

Figure 2. Testbed topology

desktop computer combines the functionality of an access point (AP) and access router (AR). All computers are equipped with IEEE 802.11b wireless interfaces. These interfaces run in ad hoc mode and are used for the transmission of payload and routing protocol packets. As routing protocol, we employ the AODV IPv4 implementation of [17]. The laptops are positioned in a way that each of them can establish a direct link to any other laptop, i.e., we have a fully-meshed network topology.

The movement of the laptops and the resulting connectivity graph is emulated: Each node moves virtually according to a mobility trace that is stored in a simple text file (ns-2 mobility file). Packets transmitted between laptops that are currently not within transmission range of each other (according to their virtual position) are discarded. This virtual dynamic topology is controlled by the desktop. Via an Ethernet connection, it informs the laptops about their current (virtual) neighbors and sends commands to set the packet filters accordingly. The actual filtering is achieved on the MAC layer using the tool iptables. The setup is implemented using the NRL mobile network emulator (MNE) [18] running on the desktop.

As the network topology is emulated while all nodes are physically linked together, the nodes share a single channel. On the one hand, this reduces the available bandwidth per node; on the other hand, hidden or exposed node problems do not occur. As we focus on the performance of the network layer, we ignore these link layer issues.

\section{Mobility Models}

There is an ongoing discussion in the research community as how to model the mobility of nodes in performance studies. Purely stochastic mobility models can be implemented quickly, and they yield "averaged" performance values, which can be easily reproduced by other 
researchers. Scenario-based studies - which include obstacles $[19,20]$ and/or use real-world mobility traces [21] - are more realistic but produce results that are only valid in this particular scenario. In this paper, we focus on purely stochastic mobility models, namely on the RWP and $\mathrm{RD}$ model. As mentioned above, we use pre-recorded mobility files to determine the movement of the nodes in the network emulation. These files have been generated from stochastic simulations of the two used models.

\subsection{Random Waypoint Model}

The most popular mobility model in current research on ad hoc networks is the RWP model. It describes the movement of a node in a bounded area as follows (see Fig. 3(a)): A node randomly chooses a destination point ("waypoint") $\mathbf{P}$ and moves with speed $V$ on a straight line to this point. After waiting a pause time $T_{p}$, it chooses a new destination and speed, moves to this destination, and so on. This movement can be described as a sequence of random variables

$$
\left\{\left(\mathbf{P}_{i}, V_{i}, T_{p, i}\right)\right\}_{i \in \mathbb{N}}=\left\{\left(\mathbf{P}_{1}, V_{1}, T_{p, 1}\right),\left(\mathbf{P}_{2}, V_{2}, T_{p, 2}\right), \ldots\right\},
$$

where an additional waypoint $\mathbf{P}_{0}$ is needed for initialization. Each destination point $\mathbf{P}_{i}$ is randomly chosen from a uniform distribution over the area. The movement from one waypoint to the next waypoint is called a movement transition. The speed as well as the pause times are chosen from an arbitrary probability distribution with well defined expected values $E\{V\}$ and $E\left\{T_{p}\right\}$, respectively.

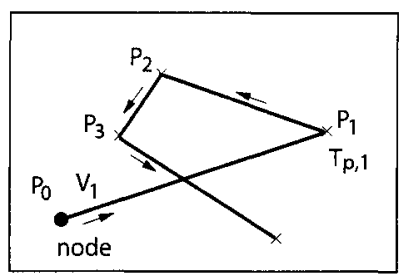

(a) RWP model

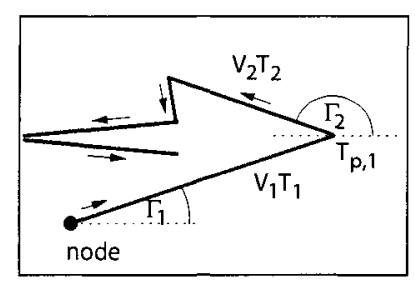

(b) RD model

Figure 3. Illustration of mobility models and random variables

Recently, it has been shown that this model has the following unintended properties:

1 Non-uniform node distribution. The probability density of a moving mobile node's location is non-uniform with a maximum oc- 
currence probability in the middle of the system area and zero occurrence probability at the very border [10, 11, 13-16].

2 Speed decay. The ensemble average of the nodes' speed decreases over time until a steady-state is achieved. If the speed $V$ is chosen between $\left[0, v_{\max }\right]$ in each waypoint, the network may converge toward a non-mobile network [22].

These properties will also influence the MRAN performance results given later in this paper.

\subsection{Random Direction Model}

In the RD model, a node chooses a destination direction $\Gamma$ (an angle) and a transition period $T$, rather than a destination point (see Fig. 3(b)). ${ }^{1}$ After moving in a straight line in the given direction for the given time, it pauses a period $T_{p}$, and then chooses a new direction, period, and speed. The complete movement process of a node can be described as

$$
\left\{\left(\Gamma_{i}, V_{i}, T_{i}, T_{p, i}\right)\right\}_{i \in \mathbb{N}}=\left\{\left(\Gamma_{1}, V_{1}, T_{1}, T_{p, 1}\right),\left(\Gamma_{2}, V_{2}, T_{2}, T_{p, 2}\right), \ldots\right\} .
$$

The directions are chosen from a uniform random distribution on the interval $[0,2 \pi[$. In this paper, the transition times are chosen from an exponential probability distribution with an expected value $E\{T\}$.

Whenever a node reaches the border of the area, it "bounces back" following the rule that the incoming angle is equal to the outgoing angle (see Fig. 3(b)). Using this border rule, a "uniform" spatial distribution of the nodes can be achieved [10].

The RD model is very popular in the research on cellular networks (see, e.g., $[9,23]$ ), but it has gained surprisingly little attention in the research community on ad hoc networking. The RWP model, on the other hand, is almost solely used in the research community on ad hoc networks. Many papers in this domain use the RWP model.

\section{Performance Evaluation}

\subsection{Setup}

The mobile nodes move independently on a square system area of length $a$. Each of them sends UDP packets with a constant bit rate $\kappa$ to the access router using multihop forwarding. All nodes have the same transmission range $r_{0}$ and equal stochastic movement parameters. The access router is located in the middle of this area.

Our performance parameters are as follows: 
- Average packet delay. The packet delay is the time that a packet needs to be transmitted from the mobile node to the access router. The average packet delay is the mean of the delays of all packets arriving at the access router. Only successfully transmitted packets are considered.

- Packet delivery fraction (PDF). The packet delivery fraction is the percentage of packets that are successfully transmitted from the mobile nodes to the AR.

To avoid transient effects, the emulation runs for a certain warm-up period. After this time, we start measuring the performance for a duration of $0.5 \mathrm{~h}$. The same experiment is repeated $\Omega$ times, each time with a different mobility trace. The parameters are shown in Table 1.

Table 1. Parameters for Performance Evaluation

\begin{tabular}{ll}
\hline Basics & $n=7$ \\
Number of mobile nodes & $\kappa=30 \mathrm{kbit} / \mathrm{s}$ \\
Constant bit rate of each node & $a=600 \mathrm{~m}$ \\
Square area with side length & $r_{0}=200 \mathrm{~m}$ \\
Virtual radio transmission range & \\
\hline Measurements & $0.5 \mathrm{~h}$ \\
Warm-up period (no measurement) & $0.5 \mathrm{~h}$ \\
Measurement period & $R=10$ \\
Number of independent experiments & $\Omega=1$ or RD \\
\hline Movement & constant $v$ \\
Speed of nodes & $T_{p}=0$ \\
Pause time & \\
RWP: uniformly distributed waypoints \\
RD: $\Gamma$ uniformly distributed \\
\multicolumn{1}{c}{$T$ exponentially distr. with mean according to (7) } \\
\hline
\end{tabular}

\subsection{Movement Parameters}

Let us now describe the parameters of the mobility models used for our performance analysis. The "level of mobility" of the RWP and RD model can be determined in terms of the nodes' speed, pause time, and direction change frequency $\lambda=\frac{\text { \# direction changes }}{\text { measurement time }}$, i.e., its expected number of direction changes per unit time. For reasonable comparison, we would like to set all parameters to be the same in both models. We set the speed of all nodes to a constant value over the entire emulation, i.e., $V_{i}=v \forall i$ for all nodes in both models. The pause time is set to zero in both models. The only parameter that needs to be determined is the direction change frequency, so that $\lambda_{\mathrm{RWP}}=\lambda_{\mathrm{RD}}$ holds. 


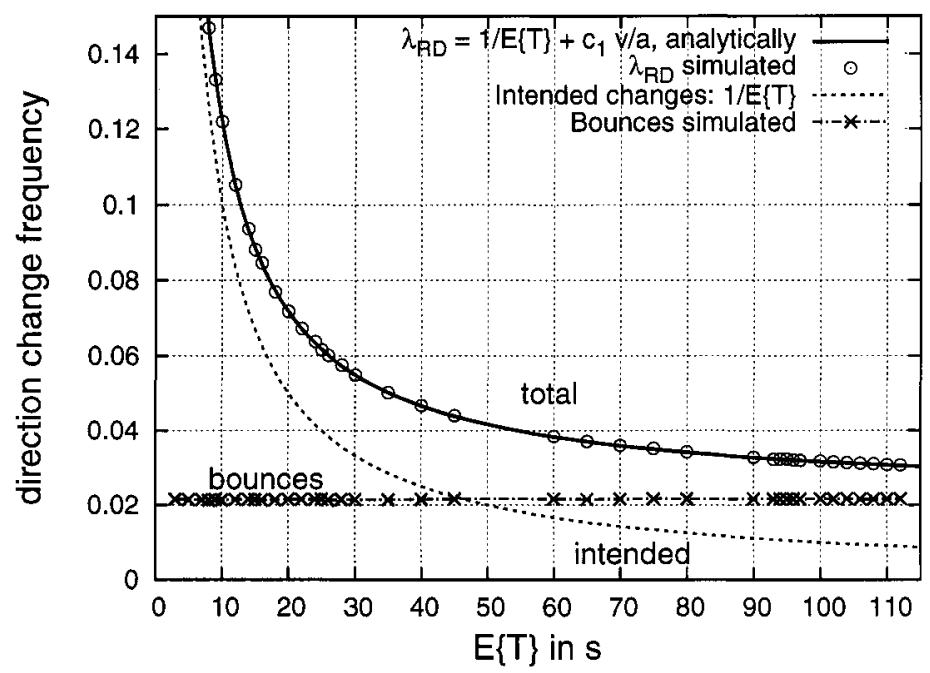

Figure 4. Direction change frequency of a mobile RD node with speed $v=10 \mathrm{~m} / \mathrm{s}$ on a square area of length $a=600 \mathrm{~m}$

Using the RWP model, the direction change frequency is

$$
\lambda_{\mathrm{RWP}}=\frac{1}{E\{T\}+E\left\{T_{p}\right\}}=\frac{1}{E\{T\}},
$$

where $T$ is the duration of one transition, i.e., the time of a movement between two waypoints. Its expected value can be written as $E\{T\}=$ $\frac{1}{v} E\{L\}$, with $L$ being the Euclidean distance between two waypoints. Since the waypoints are uniformly distributed in a square area, we can borrow well-known results from geometric probability theory [24] and write $E\{L\}=0.5214 a$. In summary, this yields

$$
\lambda_{\mathrm{RWP}}=c_{0} \frac{v}{a} \quad \text { with } c_{0}=1.918 .
$$

Using the RD model, two types of direction changes occur: (i) intended direction changes after each transition period $T$ and (ii) "forced" direction changes upon each bounce back from the border of the system area. The frequency of intended direction changes is implicitly given by $1 / E\{T\}$. The frequency of bounces is denoted by $1 / E\left\{T_{\text {bounce }}\right\}$. The total direction change frequency of the RD model is then

$$
\lambda_{\mathrm{RD}}=\frac{1}{E\{T\}}+\frac{1}{E\left\{T_{\text {bounce }}\right\}} .
$$


To determine the frequency of bounces, we make the ansatz

$$
\frac{1}{E\left\{T_{\text {bounce }}\right\}} \approx c_{1} \frac{v}{a} \text {. }
$$

The constant $c_{1}$ can be derived empirically by simulation (see Fig. 4). We obtain $c_{1} \approx 1.29$ for $E\{T\} \geq 3 \mathrm{~s}$. Note that the frequency of bounces is independent of the input parameter $E\{T\}$.

We now have sufficient knowledge to compute the value of $E\{T\}$ that must be set in the RD model, such that $\lambda_{\mathrm{RWP}}=\lambda_{\mathrm{RD}}$ is guaranteed. Combining (4), (5), and (6) yields

$$
E\{T\} \approx \frac{1}{c_{0}-c_{1}} \frac{a}{v} \approx 1.59 \frac{a}{v} .
$$

In our experiments, we measure the performance as a function of the speed $v$ for both mobility models. For each speed, we set the input parameter $E\{T\}$ of the RD model according to (7).

\subsection{Results and Interpretation}

Figure 5(a) shows the average packet delay over $v$. The vertical bars show the value range of the $\Omega$ independent experiments, i.e., the interval between the minimum and maximum average delay. We observe that the delay for the RD model is always higher than that for the RWP model. This is a consequence of the statistical spatial node distribution of the mobility models, as explained in Section 3: Since the AR is located in the middle, the number of hops between a mobile RWP node and the AR is, on average, lower than that of an RD node. This shorter hop distance results in shorter delays in the RWP model. While we did expect this qualitative behavior, the quantitative difference is indeed surprising: Independent of $v$, the RD model yields a 100\% higher average delay. This is a specific example showing that an understanding of mobility modeling is very important to interpret simulation results.

Let us analyze in more detail the causes for lower packet delays in the RWP model. The main part of the delay is caused by the route discovery process, since AODV establishes routes only on demand. We explain this in the following: A node wants to send a payload packet to the AR but has no appropriate entry in its routing table. The routing protocol will recognize that a route to the AR must be found. Hence, it buffers the packet and initiates a route discovery process by broadcasting a route request (RREQ) packet with the address of the $A R$. If the $A R$ receives the RREQ, it sends back a route reply (RREP) packet to the mobile node. When the mobile node receives the RREP, the route is established and the 


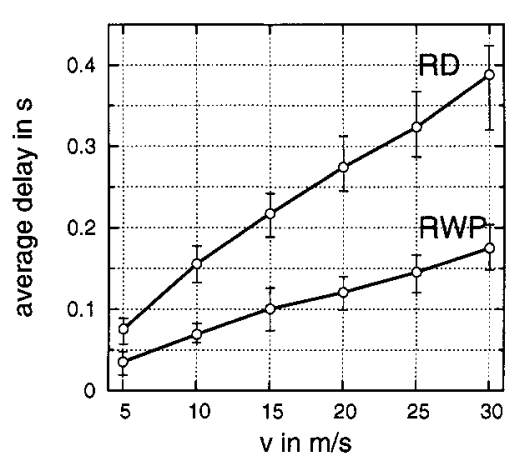

(a) Average delay

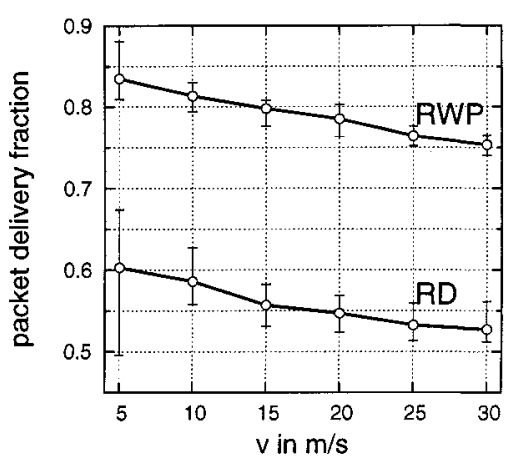

(b) Packet delivery fraction

Figure 5. Network performance over node speed $v$

payload packet can be transmitted. The duration of how long packets are buffered also depends on the number of hops between the mobile node and the AR. The reason for this is that AODV uses the expanding ring search technique for the route discovery. If an RREQ is sent for the first time, the time-to-live of this packet is set to one hop. If no RREP is received within a certain period, a second RREQ is sent with an increased time-to-life. This process is repeated until either a route is found or the maximum limit for the number of RREQs has been reached. In the latter case, no route has been found and the buffered packets are discarded.

In summary, the higher delay of the RD model is mainly caused by the on-demand nature of AODV, in particular its expanding ring search. technique. Initial measurements with a proactive routing protocol, such as OLSR [25], indicate that the packet delay is much smaller here, and the difference between the two mobility models is marginal. The reason is that proactive protocols do not buffer packets during the route discovery process.

The relation between route discovery and delay also explains the impact of speed. A higher speed of the nodes results in more frequent route disruptions, which in turn necessitates more route discoveries. Thus, the average delay of the packets is increased for a higher speed.

Finally, we analyze the packet delivery fraction (PDF), which is shown in Figure 5(b). Packets get lost either if a node can not find a route to the AR or if an established route is disrupted during the transmission of a packet. The PDF for the RD model is almost $30 \%$ lower than that for the RWP model. For example, with $v=5 \mathrm{~m} / \mathrm{s}$, the RWP model yields a reasonable PDF of $83 \%$. With the RD model, however, only $60 \%$ of the packets arrive at the AR. We conjecture that the reason for this 
behavior is as follows: at a given time instant, it is more likely in the RWP model that a mobile node finds a route to the AR. Note that this is not obvious, since the probability of a RWP node to be completely isolated is higher than in the RD model (see [26] without AR). The impact of $v$ is as follows: If the nodes move faster, routes are disrupted more frequently, and packets get lost during transmission more often. Hence, the PDF decreases for increasing $v$.

\section{Related Work}

Related work can be found in simulation-based studies on the impact of mobility modeling on performance of (stand-alone) ad hoc networks. Belding-Royer et al. [11] recognized that the RWP model causes "fluctuations" in the number of neighbors of a node, which in turn has effect on the routing performance. Bai et al. [27] did a performance study of AODV, comparing the RWP, group, freeway, and Manhattan grid mobility. Yoon et al. [22] demonstrated by simulations that the speed decay of the RWP model (Property 2) has significant impact on performance of routing protocols. The inhomogeneous node distribution of the RWP model (Property 1), has been regarded from a theoretical view in $[14,26$, $28]$; its impact on routing performance has not been elaborated so far.

Related work also comprises simulation-based studies of ad hoc networks with AODV over 802.11b using the RWP model [29]. Moreover, descriptions of other ad hoc networking testbeds can be found in $[6,30-$ $32]$ and references in [7]. Finally, performance studies of MRANs are given in $[6,33]$.

\section{Conclusions and Further Work}

Our performance analysis of an MRAN testbed has shown that we must be very careful in making statements about the (absolute) performance of a routing protocol, since it is highly sensitive to the used mobility model. In fact, understanding the impact of mobility on networklevel performance is a non-trivial task. The inherent characteristics of a mobility model have direct influence on the network topology graph, namely its static and dynamic connectivity. This connectivity, in turn, has impact on the performance of medium access control and routing. This paper has demonstrated that the routing performance difference between two common and intuitively very similar stochastic mobility models can be very high. In conclusion, we believe that the research community would greatly benefit from defining a set of "benchmark scenarios," i.e., a well-defined collection of mobility traces that can be used to evaluate new protocols. Such a collection may consist of real-world 
traces (e.g., WLAN users on a campus, cars in downtown) and purely stochastic models (such as the RWP and RD model).

\section{Notes}

1. The $\mathrm{RD}$ model described here is a generalization of the random direction model in [11]. In that paper nodes change direction only at the area border.

\section{References}

[1] J. Broch, D. A. Maltz, and D. B. Johnson, "Supporting hierarchy and heterogenous interfaces in multi-hop wireless ad hoc networks," in Proc. Workshop on Mobile Computing, (Perth, Australia), June 1999.

[2] U. Jönsson, F. Alriksson, T. Larsson, P. Johansson, and G. Q. Maguire, "MIPMANET: Mobile IP for mobile ad hoc networks," in Proc. ACM MobiHoc, (Boston, USA), Aug. 2000.

[3] Y. Sun, E. M. Belding-Royer, and C. E. Perkins, "Internet connectivity for ad hoc mobile networks," Intern. J. of Wireless Inform. Netw., vol. 9, Apr. 2002.

[4] J. Xi and C. Bettstetter, "Wireless multi-hop Internet access: Gateway discovery, routing, and addressing," in Proc. 3Gwireless, (San Francisco, USA), May 2002.

[5] R. Wakikawa, J. T. Malinen, C. E. Perkins, A. Nilsson, and A. J. Tuominen, "Global connectivity for IPv6 mobile ad hoc networks." IETF Draft, Oct. 2003.

[6] N. Bayer, B. Xu, and S. Hischke, "An architecture for connecting ad hoc networks with the IPv6 backbone (6Bone) using a wireless gateway," in Proc. European Wireless (EW), (Barcelona, Spain), Feb. 2004.

[7] E. M. Belding-Royer and C. E. Perkins, "Evolution \& future directions of the ad hoc on-demand distance vector routing protocol," Ad Hoc Netw., July 2003.

[8] D. B. Johnson and D. A. Maltz, Mobile Computing, ch. Dynamic Source Routing in Ad Hoc Wireless Networks. Kluwer, Feb. 1996.

[9] R. A. Guérin, "Channel occupancy time distribution in a cellular radio system," IEEE Trans. Veh. Technol, vol. 36, Aug. 1987.

[10] C. Bettstetter, "Mobility modeling in wireless networks: Categorization, smooth movement, and border effects," ACM Mobile Comp. Commun. Rev., July 2001.

[11] E. M. Royer, P. M. Melliar-Smith, and L. E. Moser, "An analysis of the optimum node density for ad hoc mobile networks," in Proc. IEEE ICC, (Helsinki, Finland), June 2001.

[12] C. Bettstetter and C. Wagner, "The spatial node distribution of the random waypoint mobility model," in Proc. German Workshop on Mobile Ad Hoc Networks (WMAN), (Ulm, Germany), Mar. 2002.

[13] D. M. Blough, G. Resta, and P. Santi, "A statistical analysis of the long-run node spatial distribution in mobile ad hoc networks," in Proc. ACM MSWiM, (Atlanta, USA), Sept. 2002.

[14] C. Bettstetter, G. Resta, and P. Santi, "The node distribution of the random waypoint mobility model for wireless ad hoc networks," IEEE Trans. Mobile Comput, vol. 2, July 2003. 
[15] C. Bettstetter, H. Hartenstein, and X. Pérez-Costa, "Stochastic properties of the random waypoint mobility model," ACM/Kluwer Wireless Netw., Sept. 2004.

[16] W. Navidi and T. Camp, "Stationary distributions for the random waypoint mobility model," IEEE Trans. Mobile Comput., vol. 3, Feb. 2004.

[17] "AODV-UU." http://www.docs.uu.se/docs/research/projects/scanet/aodv.

[18] W. Chao, J. P. Macker, and J. W. Winston, "NRL Mobile Network Emulator," Tech. Rep. NRL/FR/5523-03-10,054, Naval Research Laboratory, Jan. 2003.

[19] A. Jardosh, E. M. Belding-Royer, K. C. Almeroth, and S. Suri, "Towards realistic mobility models for mobile ad hoc networks," in Proc. ACM MobiCom, (San Diego, USA), Sept. 2003.

[20] J. Kammann, M. Angermann, and B. Lami, "A new mobilty model based on maps," in Proc. IEEE VTC, (Orlando, USA), Oct. 2003.

[21] J. G. Jetcheva, Y.-C. Hu, S. PalChaudhuri, A. K. Saha, and D. B. Johnson, "Design and evaluation of a metropolitan area multitier wireless ad hoc network architecture," in Proc. IEEE WMCSA, (Monterey, USA), Oct. 2003.

[22] J. Yoon, M. Liu, and B. Noble, "Random waypoint considered harmful," in Proc. IEEE Infocom, (San Francisco, USA), Apr. 2003.

[23] C. Hartmann and J. Eberspächer, "Adaptive radio resource management in F/TDMA cellular networks using smart antennas," Eur. Trans. Telecom., Sept. 2001.

[24] B. Ghosh, "Random distances within a rectangle and between two rectangles," Bull. Calcutta Math. Soc., vol. 43, 1951.

[25] T. Clausen and P. Jacquet, "Optimized Link State Routing Protocol (OLSR)." IETF RFC 3626, Oct. 2003.

[26] C. Bettstetter, "Topology properties of ad hoc networks with random waypoint mobility," in Proc. ACM MobiHoc, (Annapolis, USA), June 2003. Short paper.

[27] F. Bai, N. Sadagopan, and A. Helmy, "IMPORTANT: a framework to systematically analyze the impact of mobility on performance of routing protocols for ad hoc networks," in Proc. IEEE Infocom, (San Francisco, USA), Mar. 2003.

[28] P. Santi, "The critical transmitting range for connectivity in mobile ad hoc networks," IEEE Trans. Mobile Comput., 2004. To appear.

[29] S. R. Das, C. E. Perkins, E. M. Royer, and M. K. Marina, "Performance comparison of two on-demand routing protocols for ad hoc networks," IEEE Personal Commun. Mag., Feb. 2001.

[30] D. A. Maltz, J. Broch, and D. B. Johnson, "Lessons from a full-scale multihop wireless ad hoc network testbed," IEEE Personal Commun. Mag., Feb. 2001.

[31] D. S. J. DeCouto, D. Aguayo, B. A. Chambers, and R. Morris, "Performance of multihop wireless networks: Shortest path is not enough," in Proc. Workshop on Hot Topics in Networking (HotNets), (Princeton, USA), Oct. 2002.

[32] M. Möske, H. Füßler, H. Hartenstein, and W. Franz, "Performance measurements of a vehicular ad hoc network," in Proc. IEEE VTC, (Milan, Italy), May 2004.

[33] M. Ghassemian, P. Hofmann, C. Prehofer, V. Friderikos, and H. Aghvami, "Performance analysis of Internet gateway discovery protocols in ad hoc networks," in In Proc. IEEE WCNC, (Atlanta, USA), Mar. 2004. 